Erdbebens und der vulkanischen Tätigkeit bestritten. Wenn letztere mit Erdbeben in Zusammenhang stehe, so sei sie lediglich eine Folge der Erschütterung, d. h. die Lava könne durch die vom Erdbeben erzeugte Spalte ausfliessen, was manchmal geschehe, manchmal nicht. Pocts verwirft die Ansicht von der Periodizität der valkanischen Fraptionen und der Erdbeben sowie iiber den Zusammenhang beider mit den atmosphärischen Störungen und den kosmischen Verhältnissen. Er verweist auf die Statistik der Eruptionen und der Erdbeben, die gar keine Periodizität zeigen.

Schliesslich sei noch des umfangreichen, gut illustrierten Erdbebenbuches von Stanislads Mednier (11) gedacht, welches mit der lebhaften Scbilderung des Eindruckes beginnt, den die Nachricht von der Zerstörung Messinas in einer Pariser Familie hervorruft. Es wird uns erzählt, dass bei einem Mitglied dieser Familie, dem jungen ALвеRт Durozren dieser Eindruck ein so nachhaltiger gewesen sei, dạs er alle Nachrichten, die er über das Erdbeben von Messina und andere Katastrophen erhalten konnte, gesammelt, und sich eingehend mit den über die Ursachen der Erdbeben ansgesprochenen Ansichten beschäftigt habe. Die Resultate der Studien dieses jungen Mannes wären es, welche St. Mednien vorlegt. Offenbar hat er diese Einkleidung gewäblt mit Rücksicht darauf, dass die beiden Hauptteile des Buches "Les catastrophes contemporaines" und "L'histoire" sich im wesentlichen auf eine möglichst lebendige Schilderung der Erdbebenwirkungen beschränken und der dritte Teil "La science" in Umfang und Bedeutung wesentlich hinter den beiden ersten Teilen zurückbleibt. Dies darf kein Vorwurf für ein Werk sein, das sich an möglichst weite Kreise wendet und vor allem den Zweck verfolgt, dieselben für das $\mathrm{zu}$ interessieren, was wir gegenwärtig über die Erdbeben wissen. Als ein gutes Kompendium der Erdbebenkunde darf das MedNIER'sche Buch freilich nicht bezeichnet werden, ein solches würde aber wohl auch von den Lesern, für welche Meunier scbrieb, schon nach der Durchsicht weniger Seiten als zwar höchst wissenschaftlich und achtenswert, aber auch unwiderstehlich langweilig bei Seite gelegt worden sein, während die frische und lebendige Darstellung der Erdbebenerscheinungen und die karze, mehr durch Andeutungen als durch ausfübrliche Darlegung der bisherigen Ergebnisse der Erdbebenforschung wirkende Erörterung derselben wohl geeignet erscheint, das Interesse für die Seismologie in weiteren Kreisen wachzurufen. Aber auch der Fachmann wird das Meonier'sche Buch nicht durchsehen ohne mannigfache dankenswerte Anregungen zu empfangen.

\title{
Über die mikroseismischen Bewegungen.
}

Von H. Benndorf, Graz.

1. Hecker, O.: Über die Ursachen der mikroseismischen Bewegung. Aus ,Seismometrischen Beobachtungen in Potsdam in der Zeit vom 1. Januar bis 31. Dezember 1905." Berlin 1906.

2. KLotz, O.: Mikroseisms. Jour. of the Royal astronornical Society of Canada. 1908. p. 195.

3. Galitzin, B.: Über mikroseismische Bewegungen. Beiträge zur Geophysik 10. p. 86.1909 . 
4. SchneIdex, R.: Über die pulsatorischen Oscillationen (mikroseismische Unruhe) des Erdbodens im Winter 1907/08 in Wien. Mitt. d. Erdbeben-Kommission. Neue Folge. Nr. 35. Wien 1909.

5. LiNke, F.: Die Brandungsbewegungen des Erdbodens und ein Versuch ihrer Verwendung in der praktischen Meteorologie. Ablıandl. d. kön. Ges. d. Wiss. zu Göttingen. Neue Folge. VII. 1909.

Ausser den eigentlichen Erdbeben und den Störungen, die lokaler Natur sind (Strassenverkehr etc.) zeichnen die Seismographen auch eine eigentümliche Art kleiner Wellen anf, die unter dem Namen „mikroseismische Unruhe“, „Pulsatorische Oscillationen“, „Pendelunruhe" usw. bekannt sind. Bereits eine grössere Zahl nambafter Seismologen hat sich mit ihrom Studium eingehend beschäftigt, ohne jedoch za einer allgemein anerkannten Frklärung dieser Bewegungen gelangen zu können; um die Frage nach der Natur dieser Bewegungen und ihrer Ursachen womöglich einer Lösnng entgegen zu führen, wurde daher auf der Tagung der permanenten Kommission der internationalen seismischen Assoziation im September 1907 im Haag eine spezielle Kommission gewählt und mit dem Stadium der mikroseismischen Bewegungen beanftragt. $\mathrm{Ob}$ sie derzeit ihren Bericht bereits erstattet hat, ist dem Ref. nicht bekannt; indess sird in den letzten Jahren eine Reihe von Arbeiten über dieses Thema erschienen, die die Erkenntnis dieser Erscheinungen wesentlich gefördert haben und über die daher im folgenden referiert werden soll.

HECKER unterscheidet 4 Arten von mikroseismischer Bewegung nach den Aufzeichnungen der Potsdamer Station und zwar:

1. Bewegungen ganz kurzer Periode bis etwa 4 Sekunden:

2. Bewegungen mit einer Periode von etwa 7 Sekunden,

3. Bewegungen mit einer Periode von etwa 30 Sekunden,

4. Bewegungen mit einer Periode von einer oder mehrerer Minuten.

Die Bewegungen erster Art rühren von Erschütterungen her, die durch den Verkehr, industrielle Werke etc. hervorgerufen sind, die Bewegungen 4. Art treten zu selten auf, um vorläufig etwas über ihre Natur aussagen zu können.

Die Wellen mit 7 Sekunden Periode treten hauptsächlich im Winter auf und dauern jeweiljg einen oder mehrere Tage an; bei Untersachung ihrer Abhängigkeit von meteorologischen Faktoren in den Jahren 1904 und 1905 ergibt sich: Die 7 Sekundenwellen sind unabhängig vom lokalen Wind, dem lokalen Luftdruckgradienten und ebenso von Temperatur- and Laftdruckänderungen; dagegen lässt sich eine entschiedene Abhängigkeit von den Maximalluftdruckdifferenzen über ganz Enropa konstatieren in dem Sinn, dass steigenden Druckdifferenzen wachsende Amplituden der Bodenbewegung entsprechen; mit dem Seegange an der Norwegischen Küste verglichen, ergibt sich nur eine geringe Zunahme der Bodenbewegung mit wachsendem Seegange.

Im Gegensatz zu den 7 Sekundenwellen zeigen die 30 s. Wellen einen deutlichen Zusammenhang mit der örtlichen Windstärke und dem lokalen Luftdruckgradienten. Hecker meint daher, die 30s. Wellen seien durch die Reibung des Windes am Erdboden hervorgerufen, während er sich bezüglich der Ursachen der 7s -Wellen keiner bestimmten Ansicht anschliesst.

KLotz benutzt das Material der Erdbebenstation in Ottawa (Canada) aus dem Jahre 1907 und 1908 und kommt zu ähnlichen Resultaten wie Hecker; die 
mikroseismische Bewegung (5-6s-Wellen) ist am häufigsten und stärksten in Winter (Oktober), am geringfügigsten im Sommer (Juli, August); lokale Winde sind ohne Einfluss, ebenso Temperaturänderungen. Der einzige Zusammenhang mit meteorologischen Verhältnissen, der sichergestellt werden konnte, war der, dass wenn ein Minimum über dem St. Lorenz-Golf lagert, die mikroseismischen Bewegungen stets auftreten, besonders stark, wenn sich das Maximum südlich davon über der atlantischen Küste befindet.

Die Beobachtungen Galitziss sind in Pulkowa gemacht und beziehen sich auf Ende 1907 und Anfang 1908. Seine Resultate bestätigen die Untersuchungen Heckers vollständig; in der Dentung derselben weicht Galitzin insofern von HEcker ab, als der den 30 s - Wellen noch lokalere Ursachen zuschreibt, (Bewegung des Bodens bei Wind durch nahestehende Bäume, durch den Druck auf die Mauern der Gebäude), bezüglich der kurzperiodigen mrikroseismischen Bewegungen (4s -6 s Periode) sich der neuern Ansicht von WIECHERTs anschliesst, der annimmt, dass wir es dabei mit Eigenschwingungen der auf dem Magma ruhenden Erdkruste zu tun haben, die in erster Linie durch die Dünung des Ozeans aus. gelöst werden.

Schneroer untersucht das Material der Wiener Station Winter 1907/08 in besonders eingehender Weise; die am häufigsten auftretenden Wellen haben eine Periode von ca. 6s and ein Amplitude im Mittel von $0,003 \mathrm{~mm}$ wirklicher Bodenbewegung, die Vertikalkomponente der Wellen ist bedentend geringer als die Borizontalkomponente. SchneIder schliesst sich der Anschauung WiEcherts an und führt für sie folgende Gründe an: Lokale Winde, sowie Luftdruckverteilung sind ohne Einfluss anf die Bewegungen. Stärme auf dem atlantischen Ozean rufen nur dann starke Bewegung hervor, wenn sie einen gegen die Küste gerichteten Seegang verursachen. Schliesslich macht SchNeIDER noch den interessanten Versuch, die gleichzeitig auftretenden Bodenbewegungen in Hamburg, Strassburg und Wien zu berechnen und findet dabei eine erhebliche Abnahme der Amplitude der Wellenbewegung mit der Entfernung rom atlantischen Ozean.

Am eingehendsten beschäftigt sich Linke mit unserer Frage und der Titel seiner Abhandlung zeigt schon, dass auch er der WIECHERT schen Hypothese beipflichtet. Sein Beobachtungsmaterial stammt aus Apia (1905/06). Die Lage dieser Station mitten im grossen Ozean ermöglicht es, Zusammenhänge mit der Meeresbrandung zu untersuchen, wie es für Festlandstationen nicht in gleicher Weise tunlich ist. Seine Untersuchungen führen zu dem unzweifelhaften Resultat, dass die mikroseismischen Bewegungen (die übrigens bedeutend intensiver sind als bei Festlandstationen) in Samoa durch die Brandung hervorgerufen werden; dieser Nachweis wird besonders dadurch schlagend, dass neben statistischen Zusammenstellungen Einzeluntersuchungen der Wirkung individueller Stürme herangezogen werden.

Als Gesamtresultat der oben besprochenen Arbeiten kann vielleicht folgendes ausgesprochen werden:

Die mikroseismischen Bewegungen kurzer Periode (um 6s herum) sind unabhängig von lokalen Einflüssen der Wetterlage; notwendige, aber nicht hinreichende Bedingung für ihr Auftreten ist das Bestehen eines grossen Luftdruckminimums über dem Ozean. Von den bis jetzt aufgestellten Hppothesen vermag nur die WiEcherT sche die Beobachtungen gut zu erklären; es kann daher gegenwärtig als recht sicher gestellt angenommen werden, dass wir es bei den mikro- 
seismischen Bewegungen mit Schwingungen des Erdbodens zu tan haben, die durch die Brandungsbewegungen des Ozeans hervorgerufen sind. Welcher Art diese Schwingungen sind and wie weit sie durch die lokale geologische Beschaffenheit des Beobachtungsortes modifiziert sind, bleibt weiterer Forschung vorbehalten.

\title{
Angelo Heilprins nachgelassenes Werk über die Montagne Pelée.
}

\author{
Von A. Bergeat, Königsberg.
}

Heirrain, Argelo: The Eruption of Pelée; a summary and discassion of the phenomena and their sequels. Philadelphia 1908.

Kein anderer Vulkanausbruch hat eine so grosse Anzahl von Veröffentlichungen gezeitigt, wie derjenige der Montagne Pelée, kein anderer ist wohl, wenigstens in seinem späteren Verlaufe sorgfältiger überwacht worden. Aber die Unnahbarkeit des Katastrophenherdes und der Untergang der meisten Augenzeugen hat auch hier wiederum so manche Frage offen gelassen und trotz Lacrorx' ausführlichem Bericht muss zugegeben werden, dass gerade die beiden merkwürdigsten Erscheinungen in der letzten Eruptionsgeschichte des Berges, nämlich die Natur der heissen Sturmwolken und, wenn auch weniger, die Hervortreibung der berühmten Felsnadel noch nicht völlig aller Rätsel entkleidet sind. Jeder weitere Bericht von Augenzeugen verdient darum Beachtung.

Angero Hellpris wurde in den Maitagen des Jahres 1902 in den Tagesblättern wegen seiner mutigen Besteigungen des Vulkans viel genannt. Er war der Erste, der bald nach der Katastrophe von Saint Pierre bis an den Rand des Kraters vordrang, als dessen unheilbringende Tätigkeit noch lange nicht erschöpft war, und der über die ersten Veränderungen in ihm Kunde brachte. Er hat seitdem noch dreimal, zuletzt im Februar 1906, den Berg besucht; seine ersten Beobachtungen sind hauptsächlich in den beiden reich ausgestatteten Werken „Mont Pelée and the Tragedy of Martinique " und "The Tower of Pelée" niedergelegt. Am 17. Juli 1907 ist Hermprix verstorben. In dem vorliegenden nachgelassenen Prachtbande sind noch einmal die wesentlichen Erscheinangen der denkwürdigen Eruption zusammengefasst; in ihrer Auffassung steht Heilpris teilweise im bewussten Gegensatz zu Lacrorx' Monographie, und es lohnt deshalb aus dem Werke einiges Wesentliche hervorzuheben.

Die berühmte $\mathrm{NadeI}$ ist nach HeILPRTN wie auch nach LACRoIx' Auffassung wie ein mächtiger Pflock aus der Tiefe hervorgepresst worden. Nach seiner Meinung erhob sie sich aus dem Krater selbst und bestand aus tiefgelegenem, alten, vor der Eruption bereits festen Gesteinsmaterial. Sie war durchrissen von Spalten, durch welche Schmelzfiuss injiziert wurde und die sie wohl anch von der Basis bis zur Spitze durchzogen, denn sie rauchte manchmal wie ein hober Schornstein. Die Wurzel der Nadel verlegt HeILPRIN in sehr grosse Tiefe unter der Oberfläche des Dornes, denn sie würde sonst schwerlich den heftigen Explosionen standgehalten haben. Er hält daran fest, dass die ersten Ansätze zu dem späterhin den Berg so hoch überragenden Gebilde mindestens schon am 24. August 1902, ja, wie ihn eine Skizze seines Begleiters erkennen lässt, schon am 31. Mai 